ARTICLE

\title{
Strain wave pathway to semiconductor-to-metal transition revealed by time-resolved X-ray powder diffraction
}

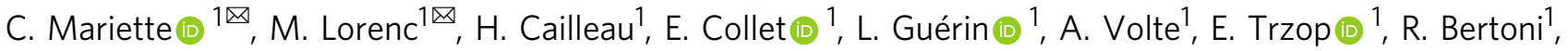

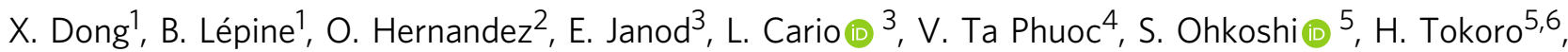

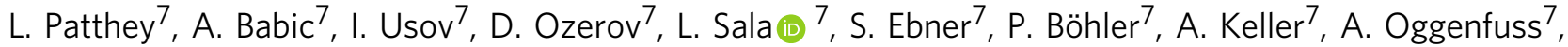 \\ T. Zmofing ${ }^{7}$, S. Redford (10 7, S. Vetter ${ }^{7}$, R. Follath (1) 7, P. Juranic ${ }^{7}$, A. Schreiber ${ }^{7}$, P. Beaud (1) 7, V. Esposito 7,10 ,

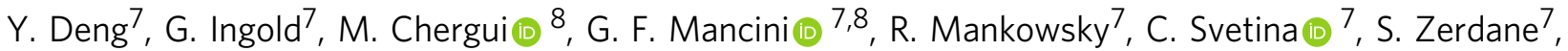 \\ A. Mozzanica ${ }^{7}$, A. Bosak ${ }^{9}$, M. Wulff ${ }^{9}$, M. Levantino (10 ${ }^{9}$, H. Lemke (i) ${ }^{7}$ \& M. Cammarata (1) ${ }^{1,11 凶}$
}

One of the main challenges in ultrafast material science is to trigger phase transitions with short pulses of light. Here we show how strain waves, launched by electronic and structural precursor phenomena, determine a coherent macroscopic transformation pathway for the semiconducting-to-metal transition in bistable $\mathrm{Ti}_{3} \mathrm{O}_{5}$ nanocrystals. Employing femtosecond powder X-ray diffraction, we measure the lattice deformation in the phase transition as a function of time. We monitor the early intra-cell distortion around the light absorbing metal dimer and the long range deformations governed by acoustic waves propagating from the laser-exposed $\mathrm{Ti}_{3} \mathrm{O}_{5}$ surface. We developed a simplified elastic model demonstrating that picosecond switching in nanocrystals happens concomitantly with the propagating acoustic wavefront, several decades faster than thermal processes governed by heat diffusion.

\footnotetext{
${ }^{1}$ Univ Rennes, CNRS, IPR (Institut de Physique de Rennes)-UMR 6251, Rennes, France. ${ }^{2}$ Univ Rennes, CNRS, ISCR (Institut des Sciences Chimiques de Rennes)-UMR 6226, Rennes, France. ${ }^{3}$ Institut des Matériaux Jean Rouxel (IMN), Université de Nantes, CNRS, Nantes, France. ${ }^{4}$ GREMAN-UMR 7347 CNRS, Université de Tours, Tours, France. ${ }^{5}$ Department of Chemistry, School of Science, The University of Tokyo, Bunkyo-ku, Tokyo, Japan. ${ }^{6}$ Department of Materials Science, Faculty of Pure and Applied Sciences, University of Tsukuba, Tsukuba, Ibaraki, Japan. ${ }^{7}$ SwissFEL, Paul Scherrer Institut, Villigen PSI, Switzerland. ${ }^{8}$ Laboratory of Ultrafast Spectroscopy, Lausanne Center for Ultrafast Science (LACUS), École Polytechnique Fédérale de Lausanne, Lausanne, Switzerland. ${ }^{9}$ European Synchrotron Radiation Facility, Grenoble, France. ${ }^{10}$ Present address: Institute for Materials and Energy Science, Stanford University and SLAC National Accelerator Laboratory, Menlo Park, CA, USA. "1Present address: European Synchrotron Radiation Facility, Grenoble, France.

凶email: celine.mariette@univ-rennes1.fr; maciej.lorenc@univ-rennes1.fr; marco.cammarata@univ-rennes1.fr
} 
$\mathrm{N}$ ew opportunities have emerged for transforming materials with femtosecond (fs) laser pulses ${ }^{1-5}$. New breakthroughs are anticipated such as attaining macroscopic transformations by driving the material on a deterministic pathway from one phase to another, very fast and efficiently. During these transformations different degrees of freedom couple sequentially and give rise to multiscale dynamics in space and time. Some of these degrees of freedom result in structural reorganizations that are crucial for stabilizing a photoexcited electronic state. Many studies have focused on optical coherent phonon oscillations around the new atomic positions ${ }^{6,7}$. However, these intracell atomic displacements preserve the crystal shape and volume. The stabilization of a new macroscopic structural order requires long-range crystal deformations that involve propagating acoustic waves. In a recent study of spincrossover molecular crystals we showed that cooperative elastic interactions help to form a macroscopically robust switched state $^{8}$. In another study of a photo-induced insulator to metal transition, it was proposed that local expansion of the lattice drives the propagation of the metallic region ${ }^{9}$. In order to examine the role of the lattice strain in photo-induced phase transition ${ }^{10}$, we investigate the semiconductor-to-metal (S-M) phase transition in $\mathrm{Ti}_{3} \mathrm{O}_{5}$ nanocrystals that exhibit even greater volume change, and a high resilience to intense laser pulses. In the aforementioned studies ${ }^{8,9}$, transient optical spectroscopy was used to follow the photo-induced changes. Unfortunately optical observables are hard to relate to structural changes, like lattice deformations. In this contribution we use X-ray pulses from an X-ray Free Electron Laser (XFEL) and synchrotrons to perform time-resolved powder diffraction, allowing a quantitative refinement of the structures on fs to $\mu$ s time scales. The ultrafast strain dynamics have been further rationalized by adopting a wellknown phenomenological elastic model ${ }^{11,12}$ and accounting for phase transformation. The highly sensitive diffraction patterns revealed the structural changes acting as precursor stress and resulting in the macroscopic phase switching that moves as a strain wave at the speed of sound, hereafter referred to as the acoustic front.

The trititanium pentoxide $\left(\mathrm{Ti}_{3} \mathrm{O}_{5}\right.$, Fig. 1) undergoes a thermal phase transition between a semiconducting (so-called $\beta$ ) and a metallic (so-called $\lambda$ ) phase around $T_{\mathrm{SM}}=460 \mathrm{~K}$ (upon heating) ${ }^{13,14}$. This S-M transition is isostructural ${ }^{15}$ (i.e., it has the same monoclinic space group $C 2 / m$ in both phases, Fig. 1a), is markedly first order and is characterized by significant changes in volume $(+6.4 \%)$ and latent (a)

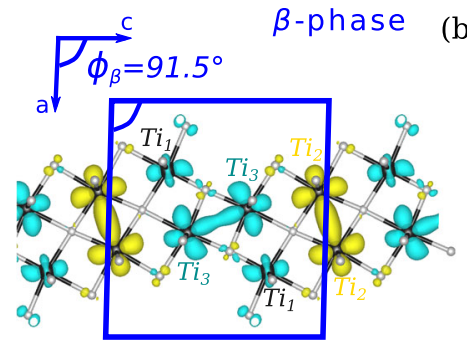

(b)

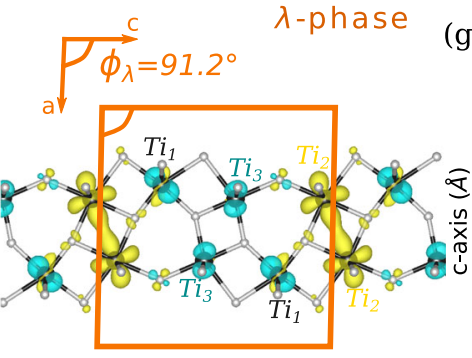

$(g)$

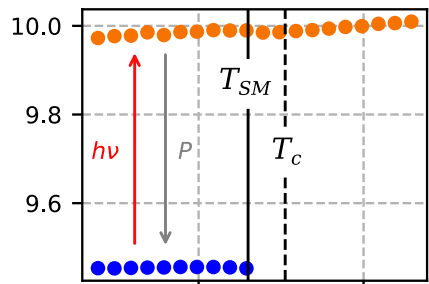

(c)

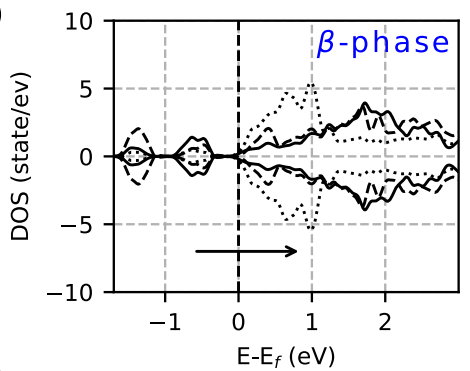

(e)

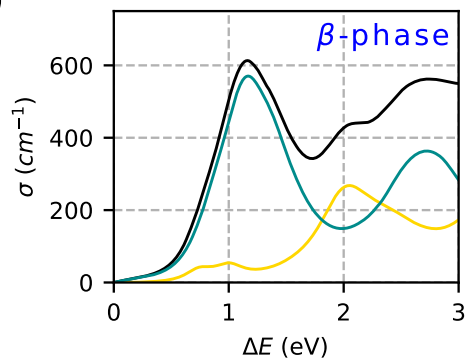

(d)

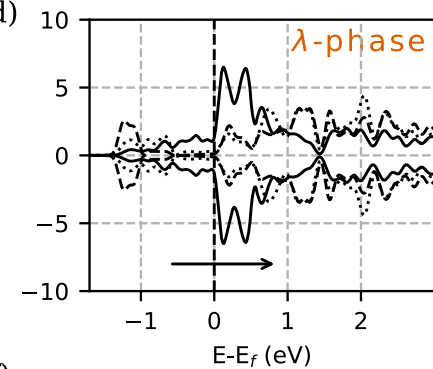

(f)

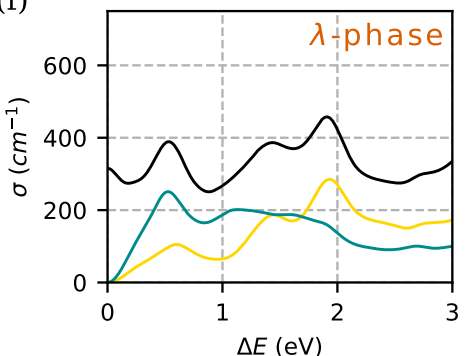

(h)

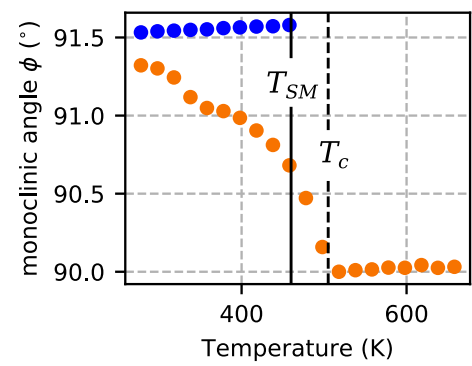

Fig. 1 Structural and optical changes upon phase transition in $\mathrm{Ti}_{3} \mathbf{O}_{5}$ nanocrystals at thermal equilibrium. $\mathbf{a}$, $\mathbf{b}$ Atomic structure of the $\beta$ - and $\lambda$ - phases, respectively, with the calculated charge density for the two bands centered at $1.1 \mathrm{eV}$ (yellow) and $0.3 \mathrm{eV}$ (cyan) below the Fermi level, strongly localized around $\mathrm{Ti}_{2}-\mathrm{Ti}_{2}$ and $\mathrm{Ti}_{3}-\mathrm{Ti}_{3}$ dimers; $\phi_{\beta}$ and $\phi_{\lambda}$ are the monoclinic angles of respectively $\beta$ - and $\lambda$ - unit cell. $\mathbf{c}$, $\mathbf{d}$ Calculated density of state projected on $\mathrm{Ti}_{1}$ (dotted lines), $\mathrm{Ti}_{2}$ (dashed lines), and $\mathrm{Ti}_{3}$ (solid lines) in the $\beta$ - and $\lambda$ - phases, respectively. The black arrow is the energy transfer corresponding to the 1.55 eV pump photons. e, f Mean calculated optical conductivity $(\sigma)$ for $\beta$ - and $\lambda$ - phases, respectively (black line). Yellow (cyan) curve is the contribution from the band at $-1.1 \mathrm{eV}(-0.3 \mathrm{eV})$. Diagonal contributions to the conductivity are shown in Fig. S1. $\mathbf{g}, \mathbf{h}$ X-ray powder diffraction data on $\mathrm{Ti}_{3} \mathrm{O}_{5}$ nanocrystals: $\mathbf{g}$ change in c-parameter of the monoclinic unit cell with a jump showing the first order phase transition at $T_{\mathrm{SM}}=460 \mathrm{~K}$ between the $\beta$ - and $\lambda$-phases; $\mathbf{h}$ Temperature dependence of the monoclinic angle $\phi$ with the first order phase transition at $T_{\mathrm{SM}}=460 \mathrm{~K}$ (between the $\beta$ - and $\lambda$-phases which co-exist in these nanocrystals below $T_{S M}$ ), and a continuous locking at $90^{\circ}$ characterizing the symmetry change towards the $\mathrm{Cmcm}$ high temperature $\alpha$-phase.

The critical temperature $T_{\mathrm{c}}=500 \mathrm{~K}$ for this second order phase transition is shown with a dotted vertical line. 
heat $\left(230 \mathrm{~kJ} \mathrm{~L}^{-1}\right)^{16}$. The increase in volume is mainly caused by expansion of the lattice along the $c$ crystalline axis. As shown in Fig. 1 the S-M phase transition involves a large intra-cell structural reorganization and the dissociation of the $\mathrm{Ti}_{3}-\mathrm{Ti}_{3}$ dimers sharing electrons on a band just below the Fermi level in the $\beta$-phase (Fig. 1a, b). From the electronic standpoint, the S-M phase transition is therefore characterized by a vanishing electronic gap related to the dissociation of the $\mathrm{Ti}_{3}-\mathrm{Ti}_{3}$ dimers ${ }^{14,17}$. A striking feature of this transition is that the metastability region of the $\lambda$-phase depends on the crystallite size. For nano-sized crystallites, the $\lambda$-phase is stable down to room temperature (RT) and below, effectively making the system bistable in a broad temperature range ${ }^{14}$. The stabilization of $\lambda$-phase is also promoted by doping ${ }^{18-20}$. In the present study, we use $\mathrm{a}_{3} \mathrm{O}_{5}$ pellet of nanocrystals with $72.5 \% \beta$ phase and $27.5 \%$ residual metastable $\lambda$ phase at RT (weight percentages, see Methods). The $\mathrm{Ti}_{3} \mathrm{O}_{5}$ nanocrystals have a typical size of about $100 \mathrm{~nm}$ and a "neat-flakes form" morphology as described in ${ }^{14}$. The crystal size was estimated from static synchrotron powder diffraction measurements performed at ESRF ID28 beamline, and described in Fig. S10. X-Ray diffraction (XRD) patterns were recorded for this pellet from 300 to $700 \mathrm{~K}$. Figure 1 panels $g$ and $h$ show the temperature dependence of the $\boldsymbol{c}$-axis parameter and the monoclinic angle $\phi$ of the different phases. As expected, the $\lambda$ and $\beta$ phases coexist from RT to $T_{\mathrm{SM}}$. The transition temperature of $460 \pm 10 \mathrm{~K}$ upon heating was determined from these XRD measurements, and it is consistent with the values previously reported for other forms ${ }^{13,14,16,21}$. At higher temperature $\left(\mathrm{T}_{\mathrm{c}}=500 \mathrm{~K}\right)$ a second order phase transition occurs towards a high symmetry metallic phase (so-called $\alpha$, of orthorhombic space group $\mathrm{Cmcm})^{13}$. This phase transition is ferroelastic: the change of crystal system from orthorhombic to monoclinic is characterized by a spontaneous shear strain. Thus the monoclinic angle $\phi$ of the $\lambda$-phase locks to $90^{\circ}$ at $\mathrm{T}_{\mathrm{c}}$. In contrast to the S-M transition, it is continuous and does not exhibit any volume discontinuity (see orange curve in Fig. 1h). The metastable $\lambda$-phase can be switched to the $\beta$ - phase by applying external pressure $\left(\mathrm{P}_{\mathrm{MS}}=0.5 \mathrm{GPa}\right.$ here, but generally strongly dependent on the morphology ${ }^{16,21}$ ). Also a photo-reversible persistent phase transition between $\beta$ and $\lambda$ phases was reported under intense ns irradiation ${ }^{14,22,23}$. Such a photoresponsive phase change material with bistability at RT is of great interest for technological applications, such as optical and heat storage ${ }^{16,21}$. The photoinduced $\beta$-to- $\lambda$ transition was also investigated in the transient regime, below the excitation threshold for permanent switching 22,24 . The full scale dynamics, from fs to $\mu$, was probed by ultrafast diffuse reflection spectroscopy on a nanogranular pellet of $\mathrm{Ti}_{3} \mathrm{O}_{5}$; those measurements were interpreted in terms of nucleation and growth process of $\lambda$ regions $^{22}$. Unfortunately the previous studies lacked either the time resolution ${ }^{24}$ or the structural sensitivity ${ }^{22}$ to observe strain wave propagation and its role in the phase transition.

\section{Results}

Time-resolved XRD. We used ultrafast XRD at the SwissFEL beamline Bernina ${ }^{25}$ to investigate the mechanism of the photoinduced semiconductor ( $\beta$ - phase) to metal $(\lambda$ - or $\alpha$ - phase) transition in a pellet of $\mathrm{Ti}_{3} \mathrm{O}_{5}$ nanocrystals. The experimental geometry is sketched in Fig. 2a. The X-ray photon energy was 6.6 $\mathrm{keV}$ and the grazing angle $0.5^{\circ}$. Considering the X-ray absorption and pellet roughness, the effective probed depth for this geometry is $z_{p}=400 \mathrm{~nm}$ (Figs. S2 and S3). The $1.55 \mathrm{eV}, 500 \mathrm{fs}$ long pump pulse impinges on the sample at an incident angle of $10^{\circ}$. At this energy, the pump penetration depth calculated from the refractive index is $\xi_{\mathrm{L}}=65 \mathrm{~nm}^{26}$. The measured time resolution is about $600 \mathrm{fs} \mathrm{FWHM}^{25}$. These studies are complemented by experiments at the ID09 beamline at ESRF, probing longer time scales with a time resolution of $100 \mathrm{ps}$ (Fig. S4). Unless stated otherwise, we will refer to the SwissFEL experiment in the following. Figure $2 b$ compares difference powder patterns taken at various time delays. The low noise, featureless transient at $-4.5 \mathrm{ps}$, shows the high quality of the data and data reduction procedures. The difference curves in Fig. $2 \mathrm{~b}$ show that major changes are already present at $500 \mathrm{fs}$ which indicates rapid structural deformations. On the picosecond time scale the difference signal increases in amplitude; the signal around some Bragg peaks, for instance (110) and (020), changes towards a characteristic "bi-polar shape", i.e. positive change towards low angles and negative change towards higher angles indicating an increase of unit-cell parameters. Broadening of some Bragg peaks is also observed ("butterfly shape"), indicating inhomogeneous lattice distortions. Such behavior is clearly observed for the isolated Bragg peak (204) from $\beta$ phase. The changes in the diffracted intensity suggest that inter-atomic reorganizations take place. The high relative intensity changes, from 10 to $30 \%$ depending on the $q$ region, indicate that large photoinduced deformations/transformations take place in the sample. Despite the complexity of the structural changes, the high-quality data allow to perform a complete Rietveld analysis for all time delays. Figure $2 \mathrm{c}-\mathrm{d}$ shows the results of the Rietveld refinement of the diffraction patterns for a reference pattern (laser OFF) and for the 7.5 ps time delay (see also Fig. S5). The full $q$ range measured and used in the refinement is displayed, namely $1.09-3.45 \AA^{-1}$. The Rietveld analysis for equilibrium and time resolved data, with unconstrained cell and atomic position parameters for the $\lambda$ - and $\beta$-phases, have similar reliability factors $\left(R_{\mathrm{p}}=3 \%\right.$ and $R_{\mathrm{wp}}=$ $7-8 \%)$. This attests the quality of the Rietveld picosecond analysis which motivates examining the evolution of the structural parameters discussed hereafter. All details about the sample, experiment, data reduction, and analysis are given in the Methods section.

Sub-ps dynamics: structural changes induced by direct optical pumping. We first describe the ultra-fast structural changes occurring within the first $500 \mathrm{fs}$ where significant intra-cell distortions are already observed. The evolution of selected distances and angles are shown in Fig. 3 for delays up to 7 ps. The typical changes are of the order of $10^{-2} \pm 2 \times 10^{-3} \AA$ and $0.5-1 \pm 0.02^{\circ}$. Comparison with the calculated electronic structure (Fig. 1a-f) helps to rationalize the observed photoinduced changes. The calculated optical conductivity is particularly instructive for describing the possible electronic transitions from the $1.55 \mathrm{eV}$ pump photons (Fig. 1e, f).

For the majority $\beta$-phase, the two bands closest to and below the Fermi level are centered at 1.1 and $0.3 \mathrm{eV}$. They have a strong $\mathrm{Ti}_{2}$ and $\mathrm{Ti}_{3}$ character, respectively. Although the $\mathrm{Ti}_{2}-\mathrm{Ti}_{2}$ and $\mathrm{Ti}_{3}-$ $\mathrm{Ti}_{3}$ dimers are both excitable above the gap by $1.55 \mathrm{eV}$ photons, the calculated optical conductivity is mainly arising from excitation from the $\mathrm{Ti}_{3}-\mathrm{Ti}_{3}$ dimers to the conduction band. The depletion of electrons in the bonding $\mathrm{Ti}_{3}-\mathrm{Ti}_{3}$ states results in a fast increase of the associated $\mathrm{D}_{33}$ distance as observed (Fig. 3b); $D_{\mathrm{ij}}$ is the distance between the $\mathrm{Ti}_{\mathrm{i}}$ and $\mathrm{Ti}_{\mathrm{j}}$ atoms. The dimers also undergo rotation as seen by the decrease in the $\theta_{332}$ angle and increase in the $\theta_{322}$ (Fig. 3a, g); $\theta_{\mathrm{ijk}}$ is the angle formed between $\mathrm{Ti}_{\mathrm{i}}, \mathrm{Ti}_{\mathrm{j}}$, and $\mathrm{Ti}_{\mathrm{k}}$ atoms. These small amplitude motions are precursor structural signatures of the isostructural phase transition from the $\beta$ - to $\lambda$ - phase. However, the rotation is smaller than for the thermal transition and thus results in a significant decrease of the $\mathrm{D}_{23}$ distance (contrary to the expected lengthening observed upon thermal transition to $\lambda$ ). On the same time scale, the bond length of the second dimer $\mathrm{D}_{22}$ is essentially constant (Fig. 3b), consistently with the fact that $\mathrm{D}_{22}$ remains essentially constant in the $\beta$ - to $\lambda$ - phase transition.

For the minority $\lambda$-phase, the photoexcitation promotes $\mathrm{Ti}_{2}$ and $\mathrm{Ti}_{3}$ electrons above the Fermi level. The contribution to the 
a)

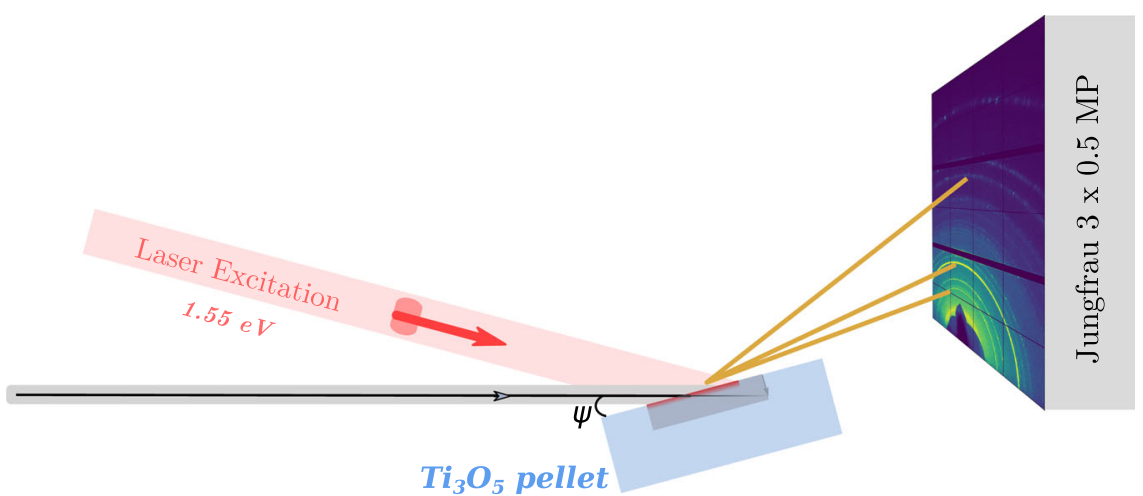

b)

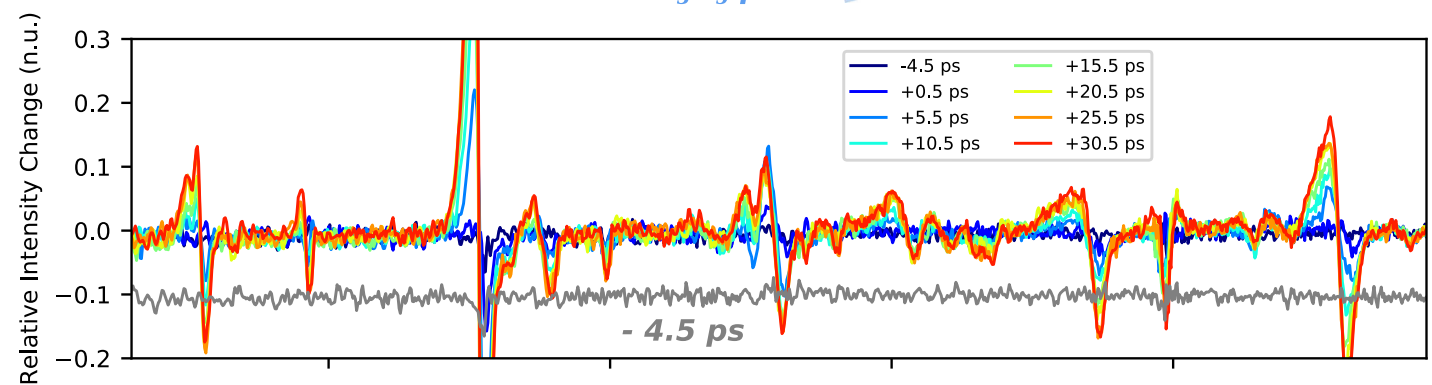

c)

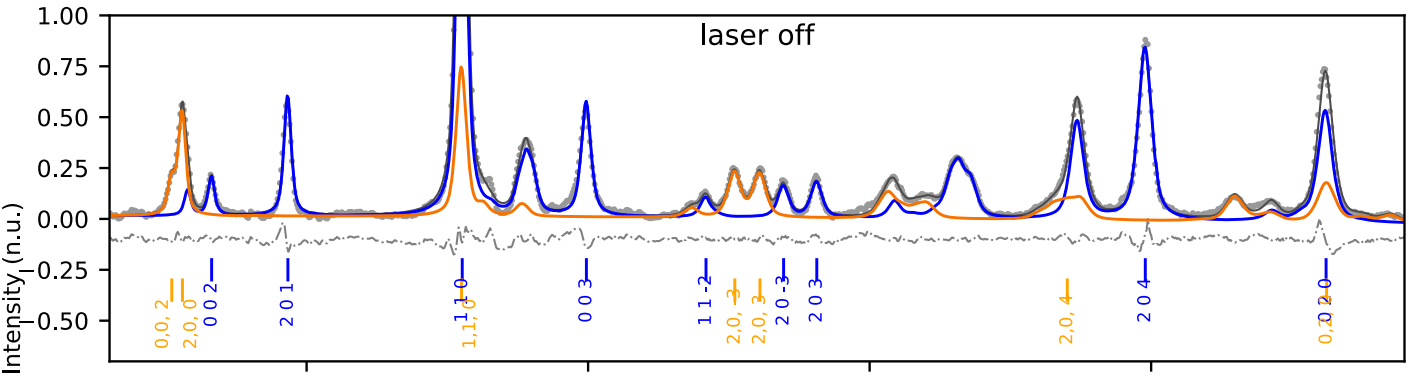

d)

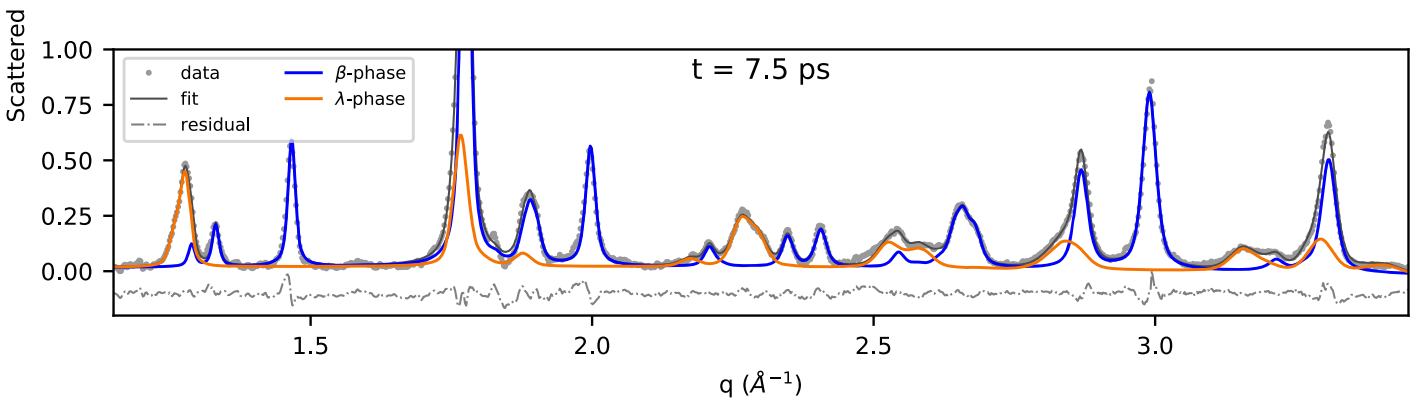

Fig. 2 Experimental setup, raw data, and structural refinement. a Experimental setup for time resolved powder X-ray diffraction in quasi grazing angle geometry. The Debye-Scherrer rings are collected on a 2D Jungfrau detector with single photon sensitivity 37 . b Difference patterns (laser on -laser $_{\text {off }}$ ) showing up to $30 \%$ variations of the signal (pump fluence $0.85 \mathrm{~mJ} \mathrm{~mm}^{2}$ ) on the ps time scale $(<35 \mathrm{ps}$ ). Gray curve shows the negative $-4.5 \mathrm{ps}$ for the non-excited delay for reference (shifted by -0.1 along y for clarity). c, $\mathbf{d}$ Rietveld refinement of reference pattern (pattern with laser off, and pattern at $t=7.5 \mathrm{ps}$ respectively). Measured powder patterns are plotted in light gray circles and result of Rietveld refinement in black, solid line. Orange and blue patterns are contributions to the refinement of the $\lambda$ - and $\beta$ - phases respectively; the residual curve is shown in gray, dashed lines.

calculated optical conductivity from the $\mathrm{Ti}_{2}$ and $\mathrm{Ti}_{3}$ electrons around $1.55 \mathrm{eV}$ is comparable (see Fig. 1h), albeit they lead to different structural effects. We observe a significant increase in $\mathrm{D}_{22}$ at the sub-ps time scale (Fig. 3e): depletion of the $\mathrm{Ti}_{2}-\mathrm{Ti}_{2}$ bonding orbital weakens the $\mathrm{Ti}_{2}-\mathrm{Ti}_{2}$ dimer, and as a result, $\mathrm{D}_{22}$ and the non-dimerized $\mathrm{D}_{33}$ become less distinguishable. In the high symmetry a phase, $\mathrm{Ti}_{2}$ and $\mathrm{Ti}_{3}$ are equivalent through a mirror plane (Fig. $3 \mathrm{~h}$ ). The relative evolution of $\mathrm{D}_{22}$ and $\mathrm{D}_{33}$ (Fig. 3e), as well as $\theta_{322}$ and $\theta_{332}$ (Fig. 3d), directly probes the ultrafast evolution of the degree of symmetry breaking in the monoclinic metallic phase. This points to a distortion towards a atomic structure.
These structural precursors appear before long-range cell deformations are observed (Fig. 3c, f). The increase of the $\lambda$ phase volume is linear as a function of time with no discontinuity at time zero; this observation had important consequences discussed in the next section. The rapid decrease in monoclinic angle (plotted in Fig. 3f) indicates that the $\lambda$ - phase undergoes ultrafast shear with partial symmetry change within 4 ps, slower than the sub-picosecond structural changes but faster than the change in unit cell volume.

Dynamics of long-range structural changes. The dynamics of the aforementioned long-range cell deformations will now be 
$\beta$ - phase

(a)
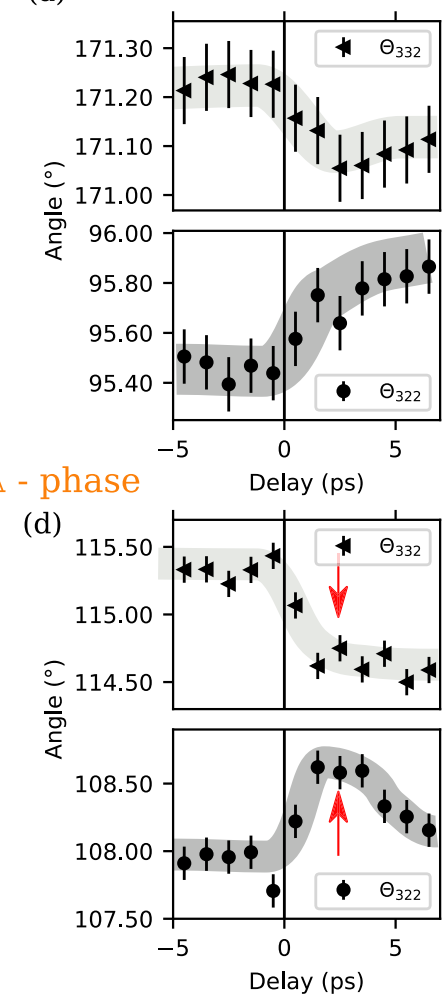

(b)

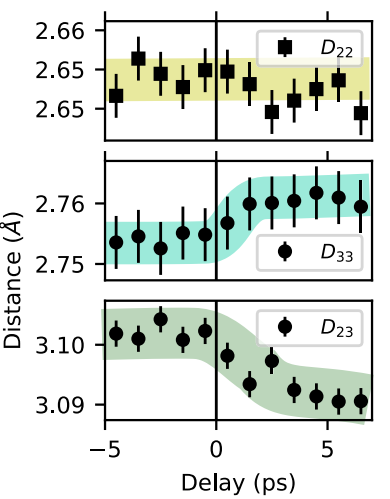

(6)

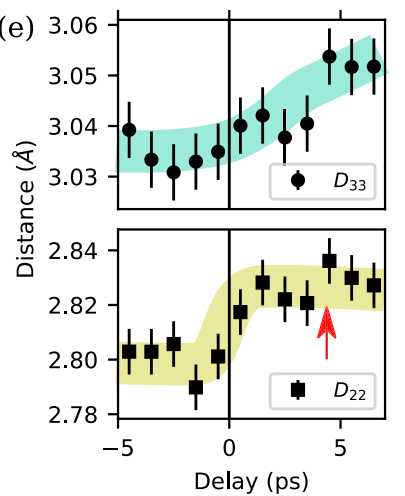

(c)

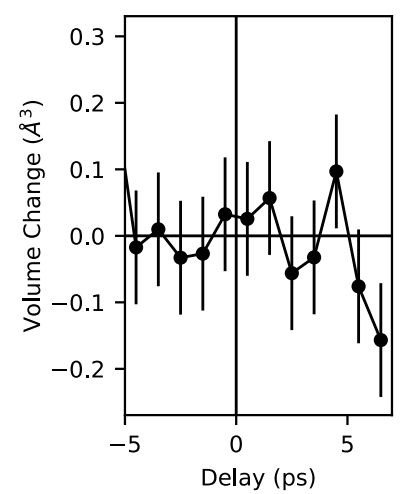

(f)

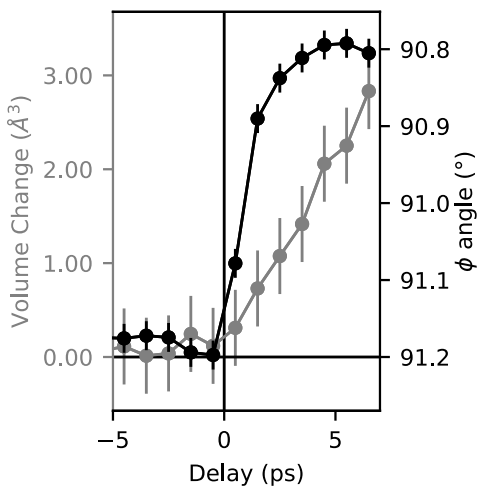

(g)

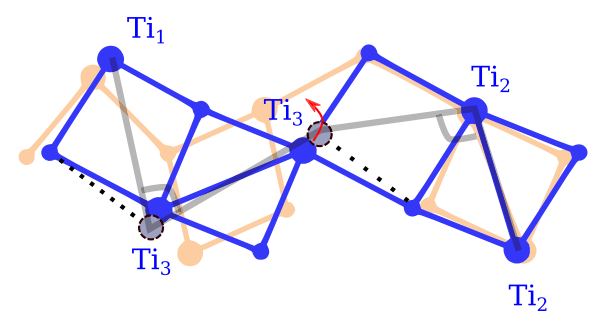

(h)

\section{$\lambda$ - phase}

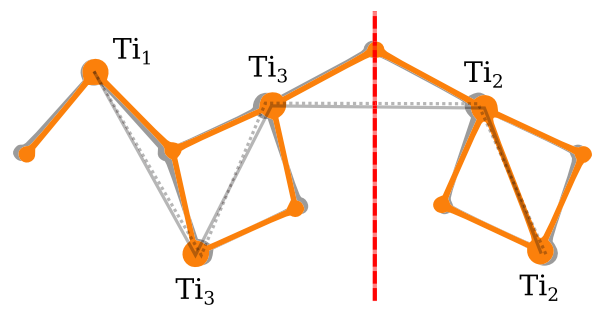

Fig. 3 Ultrafast structural changes inferred by X-ray diffraction. Evolution of important inter-atomic angles, distances, and unit cell volume changes for the $\beta$ - (a-c) and $\lambda$ - (d-f) phases ( $\phi_{i j k}=\mathrm{Ti}_{\mathrm{i}}-\mathrm{Ti}_{\mathrm{j}}-\mathrm{Ti}_{\mathrm{k}}$ angles and $D_{i j}=\mathrm{Ti}_{\mathrm{i}}-\mathrm{Ti} \mathrm{i}_{\mathrm{j}}$ distances). The evolution of the monoclinic $\phi$ angle is also reported for the $\lambda$ - phase (f). Thick transparent solid colored lines are guides for the eye. Schematic representation of the observed local distortions (exaggerated for clarity) for the $\beta$ - $(\mathbf{g})$ and $\lambda$ - $(\mathbf{h})$ phases. In $(\mathbf{g})$, the $\lambda$ - structure is represented in light orange for reference and in (h), the red line is the $m$ mirror planes lost at $T_{\mathrm{c}}$. $A$ topo of structural distortion shown in gray contour lines: dotted-before, solid-after excitation.

addressed. First the results of the Rietveld refinement will be presented followed by the discussion on a phenomenological model to explain the data.

The evolution of the $\lambda$ - phase unit cell volume takes place on the 10 ps time scale $\left(\Delta V_{\lambda}\right.$, Fig. $\left.4 \mathrm{a}\right)$. The increase is linear up to $t \approx 16 \mathrm{ps}$; In this time range a strong broadening of the Bragg peaks is also observed. It results from the strain distribution, described here by the microstrain parameter $\left(\phi_{\lambda}\right)$ as conventionally used in the analysis of powder diffraction. The evolution of the $\lambda$ - macroscopic phase fraction $\left(\Delta X_{\lambda}\right.$, Fig. $\left.4 c\right)$, signals that part of the $\beta$ - crystallites undergoes the phase transition. At $20 \mathrm{ps}, X_{\lambda}$ increases from $27.5 \%$ to $33.0 \pm 0.8 \%$ on average within the X-ray probed depth $(400 \mathrm{~nm})$. Importantly its evolution is also quasi linear up to $t \approx 16 \mathrm{ps}$, thereby the dynamics of the $\lambda$ - phase fraction follows the evolution of the $\lambda$ - phase unit cell volume. This suggests that the underlying mechanism is governed by the propagation of elastic deformations contrary to the often purported "nucleation and growth". In the latter case, the transformation can be described as a sequence of steps: after an initial formation of the seed, a relatively fast growth of the nuclei follows until the nuclei encounter boundaries that slow the process again. For this reason, such models often result in temporally sigmoidal increase of phase fractions ${ }^{27}$, which remains in stark contrast with our observation on $\mathrm{Ti}_{3} \mathrm{O}_{5}$. The experimental data suggest that the metallic $\lambda$ phase is created coherently within a traveling deformation. This motivated us to develop a strain-wave model discussed below.

The behavior of the $\beta$-phase unit cell volume is more complex. The $\Delta V_{\beta}(\mathrm{t})$ curve (Fig. $4 \mathrm{c}$ ) has a minimum around $t \approx 16 \mathrm{ps}$ whereafter the volume increases to the equilibrium value and even above. The $\beta$ - volume increases from 349.3 to $350.7 \AA^{3}$ $(+0.4 \%)$ which is rather small compared to the increase from 371.7 to $381.3 \pm 0.1 \AA^{3}(+2.5 \%)$ in the metallic phase. 
a)

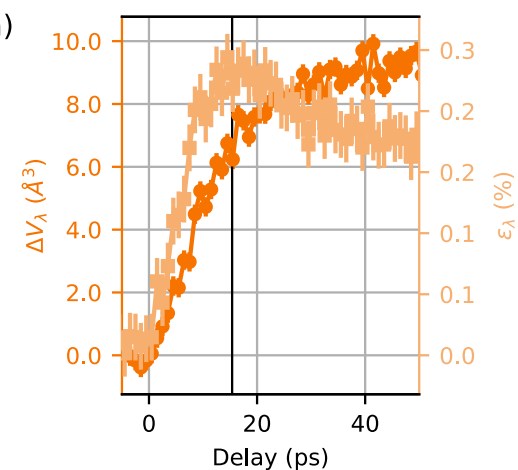

c)

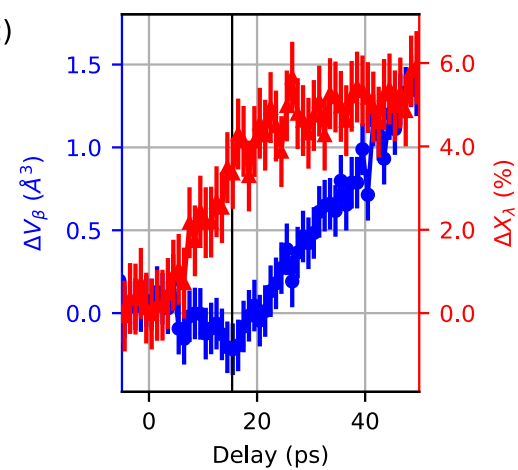

b)

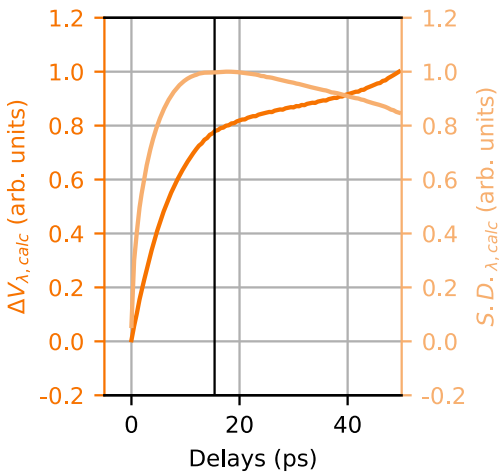

d)

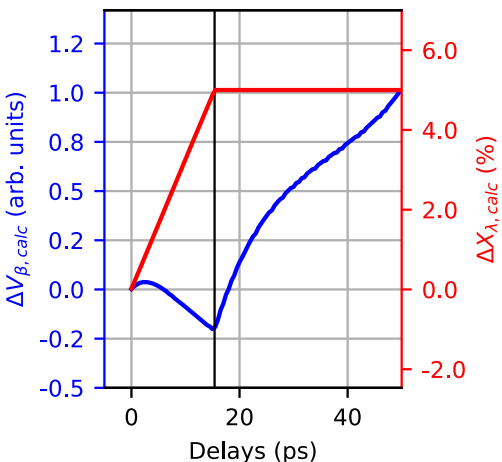

Fig. 4 Evolution of long range order and phase change on the acoustic time scales. a, $\mathbf{c}$ Parameters extracted from Rietveld refinement of the $X R D$ data. a Temporal evolution of the $\lambda$ - phase unit cell volume $\left(\Delta V_{\lambda}\right.$, dark orange, filled circles) and microstrain parameter $\phi_{\lambda}$ (light orange, filled squares). $\mathbf{c}$ Temporal evolution of the $\beta$ - phase unit cell volume $\left(\Delta V_{\beta}\right.$, blue) and $\lambda$ - phase fraction $\left(\Delta X_{\lambda}\right.$, red). Relative changes normalized to the change at $50 \mathrm{ps}$ and errors estimated as described in the Methods section. $\mathbf{b}, \mathbf{d}$ Simulation with the Thomsen mode $l^{11}$ of the parameters shown in $(\mathbf{a}, \mathbf{c})$. All details are given in the SI. $\mathbf{b} \lambda$ - crystallites. Calculation of the expected evolution of the volume change $\left(\Delta V_{\lambda}\right.$, calc, dark orange) and microstrain (S. D. $\lambda$, calc, light orange) following ultrafast excitation. Parameters of the model are taken as follows: laser penetration depth of $\chi \mathrm{L}=65 \mathrm{~nm}$, sound velocity of $v_{\mathrm{s}}=6.5 \times 10^{3} \mathrm{~m} \mathrm{~s}^{-1}$; curves normalized to the value at

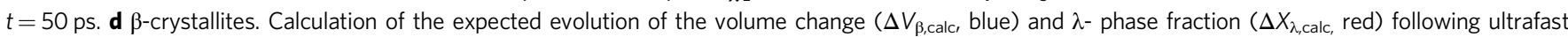
excitation. The corresponding acoustic front propagation time corresponding to $z_{\mathrm{S}}=100 \mathrm{~nm}, \phi_{\mathrm{PF}}=z_{\mathrm{S}} / v_{\mathrm{S}}$ ) is shown with solid black lines in (a, $\mathbf{b}$, $\mathbf{c}$, d).

Strain-wave model. The connection between the ultrafast photoexcitation and the strain dynamics leading to the overall volume change is described by Thomsen ${ }^{11}$ and this model has been used successfully to describe strain propagation ${ }^{12,28}$. We performed numerical calculations for the $\lambda$ - and $\beta$-crystallites separately, in order to rationalize our observations (see SI "Model calculations of Strain, Volume change and Microstrain"). In the case of a metal, the original model assumes that stress is set up by lattice heating. In the case of semiconductors, the creation of electron-hole pairs results in a more complex contribution, separated by Thomsen as electronic (proportional to deformation potential ${ }^{29}$ ) and phononic (thermal phonon, proportional to excess energy $E_{\mathrm{ph}}-E_{\mathrm{g}}$, respectively photon and gap-energy). In the present case however, the stress arises primarily from the precursor photoinduced electronic changes and the structural distortions described in the previous paragraphs, which we argue will lead to phase transition. We consider the same semi-infinite medium as in the seminal work by Thomsen ${ }^{11}$. We also assume that the initial stress profile decreases exponentially due to the absorption of the laser pulse. To establish the mechanical equilibrium between crystallites and their environment, acoustic wave packets propagate from the surface inwards, leaving behind a static deformation. Note that electronic diffusion, and how it affects the strain wave, is neglected in the model. Although this process probably occurs, it is not needed for reproducing the experimental data. The strain wave is described as follows ${ }^{11}$ :

$$
\eta(z, t)=S \times f(z, t)
$$

where $f(z, t)=\left[e^{-z / \xi}\left(1-\frac{1}{2} e^{-v_{s} t / \xi}\right)-\frac{1}{2} e^{-\left|z-v_{s} t\right| / \xi} \times \operatorname{sign}\left(z-v_{s} t\right)\right]$,
$\mathrm{S}$ is the strain at $z=0$, where the stabilized deformation is maximal. It determines the amplitude (and sign) of the volume change induced by the initial stress. The time dependence $f(z, t)$ determines the profile of the acoustic pulse, travelling at the speed of sound $v_{s}$, as well as the static deformation left behind. The speed of sound, $6.5 \times 10^{3} \mathrm{~m} \mathrm{~s}^{-1}$, was determined independently with picosecond interferometry ${ }^{30}$, sensitive to propagating acoustic wavepackets ${ }^{22}$ (Fig. S8). In the following, the time $t$ and position $z$ are related through $v_{s}$.

Based on this approach we performed numerical calculations, including the different possible responses of the two phases. The calculations qualitatively reproduce the experimental changes in lattice volume, microstrain and phase fraction as shown in Fig. 4.

For the $\beta$ crystallites, ( $75 \%$ of sample before excitation) we first consider the case where no phase transition occurs $(56 \%$ of the sample). Then, the maximum volume expansion has an amplitude $S_{\beta, \mathrm{T}} \propto V_{\beta}\left(T_{\mathrm{c}}\right)-V_{\beta}(T=300 \mathrm{~K})=1.5 \AA^{3}$. The time evolution is strictly that proposed by Thomsen (Fig. $5 \mathrm{~b}-\mathrm{d}$, upper left panel). The presence of a phase transition (19\% of the sample) increases the complexity of the model. First, the expanded region is assumed to transition to the $\lambda$ phase, and as such it no longer contributes to the strain in the $\beta$ phase (Fig. $5 b-d$, right panels). The associated stress leads to an increase in volume expressed as $S_{\beta, \text { PT }} \propto V_{\lambda}(T=300 \mathrm{~K})-V_{\beta}(T=300 \mathrm{~K})=20 \AA^{3}$ (see Fig. S7), and a significant compressive wave is launched (Fig. 5b, c). Hence the second consequence: the volume shrinkage of the $\beta$ phase near the phase front, as observed experimentally, see Fig. $4 \mathrm{~d}$. The transformation stops at $\left(t=\tau_{\mathrm{PF}}, z=z_{\mathrm{s}}\right)$, yet the strain wave 
a)
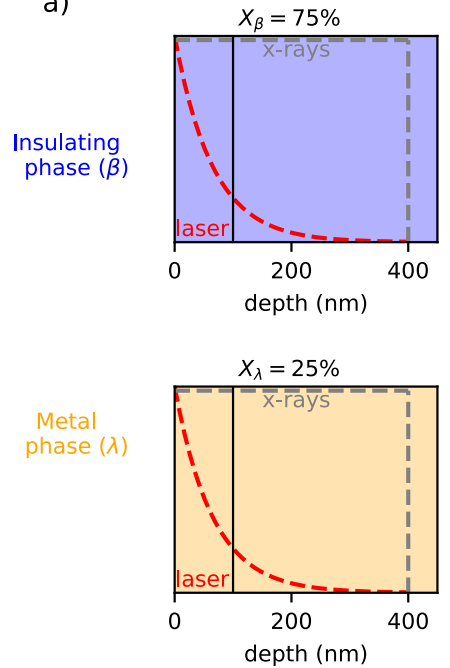

b)
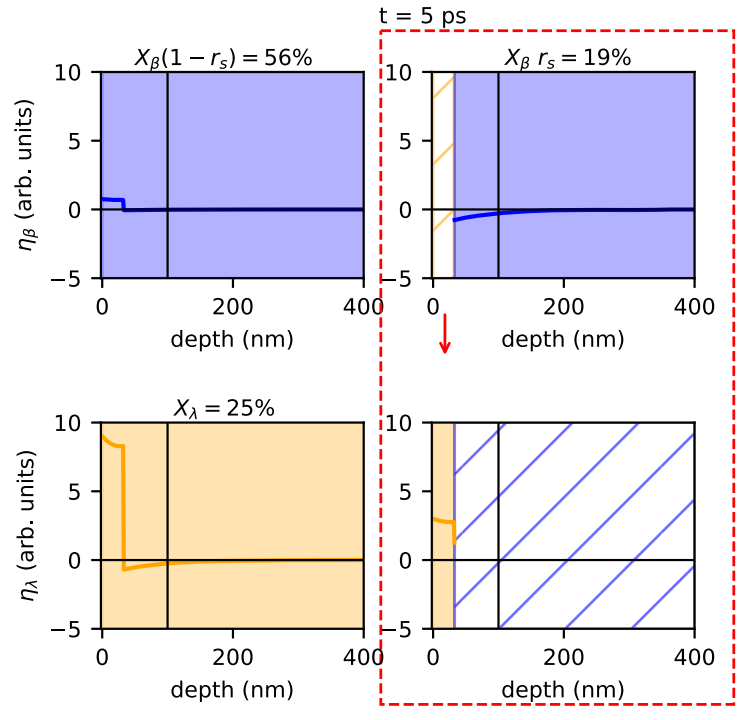

C)

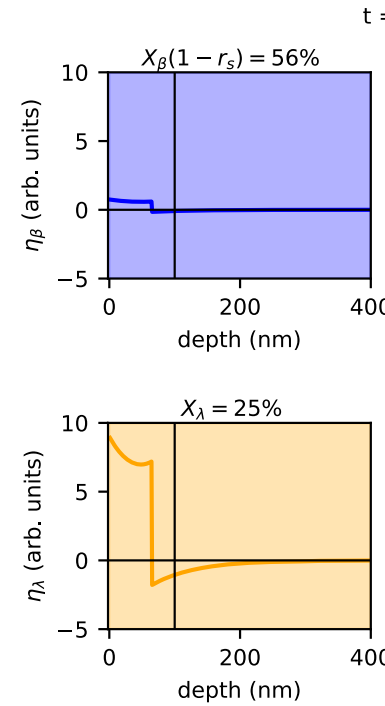

Mean crystallite size $=100 \mathrm{~nm}$ Laser penetration $=65 \mathrm{~nm}$ X-ray penetration $=400 \mathrm{~nm}$

$=40 \mathrm{ps}$

d)

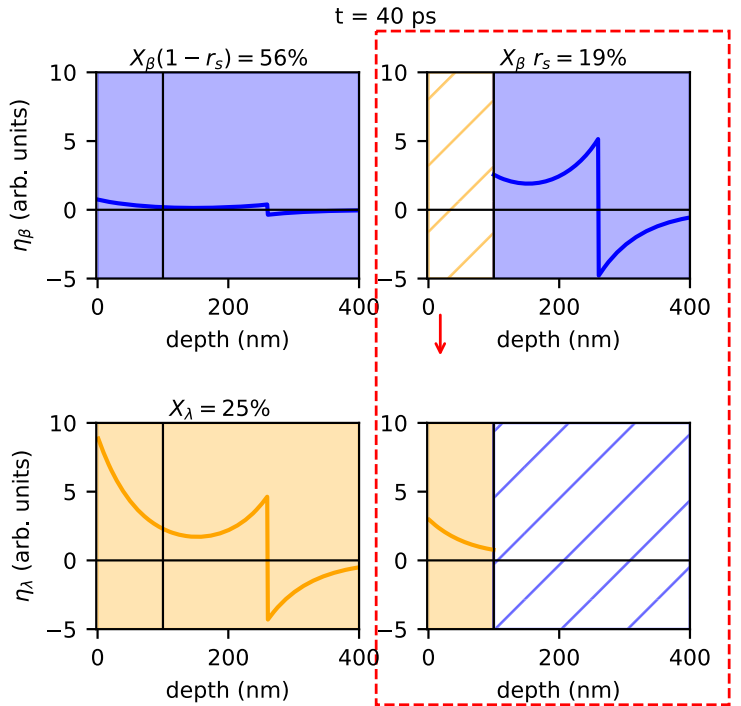

Fig. 5 Strain wave model with phase transition. The spatial dependence of the elastic strain at different times (tagged top of each panel), for $\beta$-phase crystalites (in blue, initial fraction $\left.X_{\beta}=75 \%\right)$ remaining in the initial phase (56\%, left side on each panel) and transitioning to $\lambda$ (19\%, right side on each panel), $\lambda$-phase crystallites (in orange) remaining in the initial phase (initial fraction $X_{\lambda}=25 \%$, left side on each panel) and newly formed from $\beta$ (19\% with transformation rate $r_{\mathrm{s}}=0.26$, right side on each panel). To distinguish the strained-transitioning crystalites from those strained-non transitioning, the former situation is contoured on each panel with a red dashed line and red arrow points to the transition direction. The solid vertical line at $z=100 \mathrm{~nm}$ on all panels delimits the phase-transition zone. Semi-infinite 1D medium approximates the real sample and the experimental conditions. a Initial photoinduced stress following the laser absorption profile (in red). X-ray probe depth is indicated in gray (details given in Fig. S9). The inset shows the most important values in the experiment. b-d Strain profile $\eta(z, t)$ as calculated according to the model described in the main text. For transitioning crystallites (contoured dashed red) the strain due to the transition is calculated separately for the $\lambda$ - and $\beta$-phase.

generated at $t=0$ continues to travel into the region $z>z_{\mathrm{s}}$ of the $\beta$ phase and induces a positive strain therein.

Even though we cannot distinguish experimentally between transitioning and non-transitioning $\beta$ crystallites, the respective contributions $S_{\beta, \mathrm{PT}}$, and $S_{\beta, \mathrm{T}}$ determine the sign and amplitude of the average negative change of volume observed at $\tau_{\mathrm{PF}}$. The amplitude of $S_{\beta, \text { PT }}$ is $\sim 13$ times greater than that of $S_{\beta, \mathrm{T}}$ as shown above, in agreement with the observation (Fig. 4c, d). However, any ratio in the range $\mathrm{e}^{10-20}$ would still give reasonable agreement.

For the $\lambda$ crystallites, we first consider the lattice heating from the photo-excitation of the $\lambda$ crystallite (25\% of sample before excitation). For the calculation of the average strain in this phase, we also have to include the new $\lambda$ crystallites transitioned from $\beta$. The two contributions $S_{\lambda, \mathrm{PT}}$ and $S_{\lambda, \mathrm{T}}$ are both of phononic origin and scale with the energy deposited in the lattice. For the new $\lambda$ phase, the energy transferred to the phonon bath is equal or less than $E_{p h}-E_{g}-Q=0.55 \mathrm{eV}\left(E_{\mathrm{g}}=\right.$ $0.7 \mathrm{eV}$, latent heat $Q=230 \mathrm{~kJ} \mathrm{~L}^{-1}$ as reported in ${ }^{16}$, corresponding to $\left.0.25 \mathrm{eV} / \mathrm{dimers}\left(\mathrm{Ti}_{3}-\mathrm{Ti}_{3}\right)\right)$. In the excited pre-existing $\lambda$ phase, the full photon energy $(1.55 \mathrm{eV})$ is assumed to be transferred to the phonon bath. The calculations presented in Figs. 4 and 5 are performed with the amplitude of $S_{\lambda, \mathrm{PT}}=$ $1 / 3 S_{\lambda, T}$, however, the variation of the relative contributions in the range [0-0.5] would still give a reasonable agreement.

It is important to explicitly mention that in order to limit the number of adjustable parameters and keep the discussion of the model as simple as possible, we have made the following assumptions: 
Nanocrystals are considered somewhat independently from one to another except along the depth direction.

Each nanocrystal is assumed to be monophasic before photoexcitation

Each nanocrystal is assumed to be either fully transformed after the transit of the strain wave or not transformed at all.

These hypotheses seem reasonable based on our present knowledge. In the strain model, this choice was made for the sake of simplicity as they allow to describe the entire sample as "weighted sum" of three contributions that are described below in details: $\lambda$ nanocrystals for which excitation translate into lattice heating, $\beta$ nanocrystals that transform into $\lambda$ and $\beta$ nanocrystals that remain in the $\beta$ phase and are simply heated up by the photon absorption.

An alternative hypothesis would consider a mixture of $\beta / \lambda$ domains in the crystallites before and after transformation. In this case, one would need to consider an extra compression due to the transition on the untransformed domains. Calculations including this extra contribution are displayed in Fig. S11, showing no major difference with the evolutions displayed in Fig. 4b, d.

Details of the calculations of the parameters shown in Fig. $4 \mathrm{~b}, \mathrm{~d}$ are given in SI. The strain gradient, arising from the initial stress distribution, is quantified by the standard deviation of the calculated strain (Fig. 4b). It is then compared with the observed Bragg peak broadening, quantified in the Rietveld refinement by the microstrain parameter $\varepsilon_{\lambda}$ (Fig. 4a).

\section{Discussion}

This simple model is an attempt to generalize the Thomsen model to include a phase transformation. This is essential for systems where the volume change associated with the transition is comparable (or higher) that the laser induced thermal expansion included in the original model. For the $\beta$-phase, we accounted for a partial transformation of the crystallites, and the associated change in the unit cell volume. The initially excited $\beta$-crystallites are assumed to be promptly transformed to $\lambda$ - phase concomitant with the moving acoustic wavefront. This model has its limits, most notably the feedback mechanism between volume change and transition is not rigorously solved by the model. In spite of that, the physical picture of a phase front moving with the acoustic front agrees well with the experimental data. The phase front is assumed to stop at $z_{\mathrm{s}}=100 \mathrm{~nm}$, matching the average crystallite size as measured by XRD. The acoustic front takes $\tau_{\mathrm{PF}}$ $\sim 16$ ps to travel this distance (see the black line in Fig. $4 \mathrm{a}-\mathrm{d}$ ). This is exactly when the $\beta$-phase volume shows a well defined minimum, a direct consequence of the compression exerted by the high volume of the layer that transforms to the $\lambda$-phase (Fig. 5). The latter also influences the calculated mean strain in the $\lambda$-phase (Fig. 4b). The $\lambda$-phase fraction is plotted in Fig. $4 \mathrm{~d}$ in agreement with the behavior in Fig. 4c. As a result, the 5\% average increase within the X-ray probed depth of $z_{\mathrm{p}}=400 \mathrm{~nm}$, amounts to $\sim 26 \%$ within $z_{\mathrm{s}}=100 \mathrm{~nm}$, where the phase front stops (see Fig. S9). Since the laser penetration, average crystallite size, and the phase-front breakdown limit are all about $100 \mathrm{~nm}$, we cannot speculate on the exact nature of the transition limit. Only a quarter of the crystallites undergoes transition by the strain wave, which we tentatively assign to residual static stresses pre-existing in the sample ( $\lambda / \beta$ coexistence) and/or to the random orientation of crystallites. The latter would be related to the predominant role of expansion along the $c$-axis to stabilise the new phase. Finally, the exact origin of the phase transition requires further information about the nature of the $\lambda$ - $\beta$ interface. Our study probes the bulk of volume penetrated by the X-rays, so we cannot claim that the transition at the interface is driven by the strain alone. Yet, the strain and the phase transition propagate at the same velocity showing that the strain wave determines the pathway of a truly macroscopic semiconductor to metal transition in nano materials that exhibit a volume change during the phase transition. Relying on the same physical picture, the 4 ps shear dynamics described above, would correspond to propagation on $10 \mathrm{~nm}$ with a transverse acoustic velocity of $3 \times 10^{3} \mathrm{~m} \mathrm{~s}^{-1} 16$. High resolution single crystal studies of the same compound performed at ID28 (ESRF), suggest the presence of relatively dense stacking faults along the $c$ axis (Fig. S6). Ferroelastic domains of similar size were also recently reported for an archetypal oxide ${ }^{31}$.

On longer time-scales $\left(t>\tau_{\mathrm{PF}}\right)$, the temperature and strain distributions persist until heat diffusion equilibrates the temperature over the probed area ${ }^{11,12}$. This was investigated by XRD with 100 ps time resolution at the beamline ID09 at ESRF ${ }^{32}$ (Fig. 6). The slow process can be clearly distinguished because it develops until 100 ns. The fraction of transformed $\beta$ - crystallites reaches $30 \%$ in the probed volume (see Fig. S9). The 100 ns value is consistent with expected time for heat diffusion over $D \approx 200 \mathrm{~nm}$ (in a bulk material with thermal diffusivity of $\left.230 \mathrm{~nm}^{2} \mathrm{~ns}^{-1}\right)^{16}$. The complete recovery of thermal equilibrium with the environment is observed on a $10 \mu$ s time scale, in agreement with ${ }^{24}$.

This work offers a new perspective for ultrafast control of materials with evidence of a strain wave pathway for achieving a phase transition in nanocrystallites excited by a light pulse. The key role of the long-range lattice deformation is highlighted, and so are the benefits of direct structural probes for visualising thereof. We demonstrate that state-of-the-art XFEL sources permit the probing of the interatomic motions and lattice distortions in real time, even in a bi-phasic polycrystalline powder. Our experiment, corroborated with a phenomenological elastic model, reveals unambiguously that a phase front starting from the surface is moving with sonic speed into the bulk. This process, restoring mechanical equilibrium, takes place on an ultrafast timescale and clearly precedes the much slower thermal diffusion homogenization. XFEL data suggests that the transition propagates coherently, as opposed to random growth induced by incoherent thermal kinetics (observed with synchrotron pulses on $100 \mathrm{~ns}$ time scale). Indeed nucleation-and-growth models often result in a sigmoidal temporal dependence of the new-phase fraction $^{27}$, which is markedly different from the growth we observed. The simultaneous change of the $\lambda$-phase fraction and its volume substantiate our interpretation.

We believe that the strain wave pathway is likely to be valid in a variety of other volume changing materials. Notably, the strain

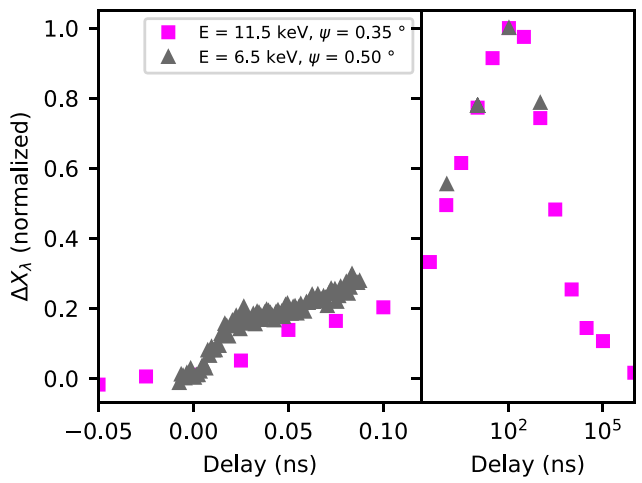

Fig. 6 Thermal propagation in the bulk pellet. Multiscale evolution (from ps to $\mathrm{ms})$ of the normalized $\lambda$ fraction $\left(\Delta X_{\lambda}\right)$ as extracted from time resolved XRD measurements at the ESRF beamline ID09 (magenta squares, $X$-ray energy $E=11.5 \mathrm{keV}$, incident angle $\psi=0.35^{\circ}$ ). The fractional change is normalized to the maximum value for allowing a direct comparison with the evolution observed at SwissFEL Bernina (gray triangles). Long time delays are presented on a logarithmic time scale. 
waves are launched directly in the material by internal precursor stresses that store mechanical energy. This is a step forward for self-contained transformation, in contrast to previously reported phase front propagation which required a hetero-structures for launching where the strain waves are created at the interface of a strongly absorbing transducer film into the phase change film $^{33,34}$. We show that our approach is nondestructive, and opens up fascinating prospects for an advanced degree of strain control through electronic and structural precursors.

\section{Methods}

Static powder laboratory XRD-temperature measurements. The static powder $\mathrm{XRD}$ as a function of temperature was measured in Debye-Scherrer geometry on a Bruker AXS D8 Advance (Mo- $\mathrm{K}_{\alpha}$ radiation selected with a focusing Göbel mirror) equipped with a MRI high temperature capillary furnace and a high-energy LynxEye detector. The flake form $\mathrm{Ti}_{3} \mathrm{O}_{5}$ powder sample ${ }^{14}$ was sealed in a quartz capillary of $0.3 \mathrm{~mm}$ in diameter.

DFT/optical properties calculations. All calculations have been carried out by using the Quantum ESPRESSO package within the framework of DFT $+\mathrm{U}$, with the Perdew-Burke-Ernzerhoff generalized gradient approximation to describe the exchange-correlation functional. Both Projector Augmented Wave basis and normconserving pseudo-potentials. The Monkhorst-Pack grid of $8 \times 8 \times 4$ in the reciprocal space was used for the Brillouin zone sampling for both $\lambda$ - phase and $\beta$ phase. The total energy of the system converged to less than $1.0 \times 10^{-6} \mathrm{Ry}$. Electronic wave functions were represented in a plane wave basis up to an energy cutoff of at 90 Ry. Crystallographic structures were taken from ${ }^{14}$. Ferromagnetic and antiferromagnetic (AFM) orders were considered. AFM order was found to be the ground-state for both $\lambda$ - and $\beta$-phases. The optical properties were computed using epsilon.x post-processing tool of the Quantum Espresso package, at the independent-particle approximation level. Both intraband and interband contributions were considered. Calculated optical density shown in Fig. 1 is the average of the 3 diagonal elements (Fig. S1)

Samples preparation. Flakes-form $\lambda$-phase samples were obtained following the synthesis method described in ${ }^{14}$. Pellets were made from flakes powder using a uniaxial press at $3 \mathrm{GPa}$. The resulting pellets have a density of $3.2 \mathrm{~g} \mathrm{~cm}^{-3}$ (a ratio of 0.8 compared to single crystal, measured by X-ray absorption). They contain a mixture of pressure-induced $\beta$-phase crystallites and $\lambda$-phase crystallites due to residual stress. The absolute $\lambda$-phase fraction is determined by Rietveld refinement around $25-30 \%$ (depending on the pellet), in good agreement with the ratio usually observed with this method ${ }^{14}$. Whether $\beta$ - and $\lambda$-could coexist within a single grain remains an open question and cannot be answered with our XRD measurements.

Experimental details-time-resolved experiment. Experiments were performed at ESRF Synchrotron (Grenoble, France) using the ID09 time resolved beamtime (exp1) and at SwissFEL (PSI, Villigen, Switzerland) at the Bernina beamline (exp2). The latter was part of the very first commissioning experiment of the entire facility.

EXP1: ESRF experiment detail. The ID09 setup consists of fast rotating choppers to select to isolate X-ray single pulses (each $100 \mathrm{ps}$ long) at $1 \mathrm{kHz}$ repetition rate ${ }^{32}$. A fast shutter was used to lower the frequency to $10 \mathrm{~Hz}$. The X-rays were partially monochromatized by using a $\mathrm{Ru} / \mathrm{B}_{4} \mathrm{C}$ multilayer monochromator resulting in $1.5 \%$ bandwidth centered at $11.5 \mathrm{keV}$ and focused by a toroidal mirror to a size of $0.1 \times$ $0.06 \mathrm{~mm}^{2}$. In order to reduce the X-ray beam footprint on the sample, the last slits $(\sim 0.6 \mathrm{~m}$ from the sample) were closed vertically to a $0.03 \mathrm{~mm}$ gap. A synchronized laser $(800 \mathrm{~nm})$ at $10 \mathrm{~Hz}$ was used to excite the sample using a perpendicular geometry configuration, with the laser hitting the sample from the top. Laser Beam size was $10 \times 0.23$ or $4.6 \times 0.21 \mathrm{~mm}^{2}$ depending on power densities (respectively below and above $1 \mathrm{~mJ} \mathrm{~mm}^{-2}$ ). Diffracted X-rays were integrated on a Rayonix MX170-HS CCD detector and azimuthally integrated using pyFAI ${ }^{35}$.

EXP2: SwissFEL experiment. The experimental setup is shown in Fig. 2a. The SwissFEL produces short X-ray pulses (estimated duration $300 \mathrm{fs}$ ) that are sent directly to the Bernina beamline ${ }^{25}$. Since the experiment was performed as part of the commissioning for the facility, the X-ray photon energy was limited to $2.2 \mathrm{keV}$. The third harmonic $(6.6 \mathrm{keV})$ was used to probe the structural changes after photoexcitation. The energy per pulse was $\sim 0.2 \mathrm{~mJ}$ and the third harmonic content of about $1 \%$. The fundamental was suppressed using absorbers. We estimate about $10^{9} 3 \mathrm{rd}$ harmonic photons per pulse at the sample position. The X-rays were focused by a Kirkpatrick-Baez mirror system. The spot was set to $\sim 200 \times 7.5 \mu \mathrm{m}^{2}$ $\left(\mathrm{FWHM} \mathrm{h} \times \mathrm{v}\right.$ ). Because of the small grazing incidence angle of $0.5^{\circ}$ the small vertical spot was essential to minimize the footprint on sample which with a length of $0.86 \mathrm{~mm}$ footprint is still one of the major contributions to the peak broadening.
The pump laser pulses, generated by a Coherent CPA amplifier (Legend) were purposely stretched to $0.5 \mathrm{ps}$ to limit the peak intensity on the sample increasing the overall time resolution to $\sim 600 \mathrm{fs}$. Note that no jitter correction ${ }^{36}$ was used for the experiment. The measured instrumental time resolution of $350 \mathrm{fs}^{\mathrm{FWHM}}{ }^{25}$ increased due to the pump pulse stretching to about $600 \mathrm{fs}$. The laser beamsize at the sample position was $300 \mu \mathrm{m}$ (FWHM). With an incident angle of $10^{\circ}$ the resulting footprint was $300 \times 730 \mu \mathrm{m}^{2}$. The repetition rate of the laser was set to 5 $\mathrm{Hz}$ minimizing residual heating effect.

Data reduction/correction. Diffracted X-rays were measured (for every single pulse) by the Jungfrau pixel detector ${ }^{37}$. The detector was calibrated to convert ADU directly in equivalent $\mathrm{keV}$ photon energy. Most pixels $(>70 \%)$ had zero counts, the others detected either one elastic photon $(\sim 6.6 \mathrm{keV}$ reading, about $3 \%$ of the pixels), a Ti fluorescent photon (centered around $4.4 \mathrm{keV}, 11 \%$ of the pixels) or a combination of the two ( 1 elastic +1 fluorescence photon). By eliminating the fluorescence photons from each image a lower background image was obtained. Each "fluorescence corrected" image has been azimuthally integrated to obtain 1D curve $^{35}$. Each data point was collected by acquiring 500 images (250 with pump laser and 250 without pump laser). The two sets were averaged (after "fluorescence correction" and azimuthal integration) to provide two curves (laser off and on). To correct for drifts in photon energy each "off image" was used to extract the average photon energy during collection of a given time delay. The corresponding "laser on" image was treated using the optimized X-ray photon energy. To further verify the stability of the data collection and correction strategy, every 10 delays a reference time delay was acquired to be sure that no drifts or permanent sample change had happened. Each powder pattern was normalized to the intensity of the air scattering contribution dominating at low scattering vectors (between 0.7 and $0.9 \AA^{-1}$ ). All raw data and data reduction scripts will be made available.

Data analysis (Rietveld refinement). Rietveld whole powder pattern profile refinements were performed following a fundamental parameter approach using the TOPAS software ${ }^{38}$. Pawley refinements were also used and both methods gave consistent evolutions of the unit cell parameters and scales factors. In both cases, refinement was performed using the $q$ range from 1.09 to $3.45 \AA^{-1}$, corresponding in this case to diffraction angles from $19^{\circ}$ to $63^{\circ}$. The profiles were described using a beam energy of $6.5 \mathrm{keV}(\lambda=1.899 \AA)$ and a gaussian emission profile with $1.3 \%$ FWHM. The peak width and sample displacement were described using the expressions from ${ }^{39}$, vertical width and incident angle of the beam being fixed as measured $\left(4 \mu \mathrm{m}\right.$ and $0.5^{\circ}$ respectively). The sample detector-distance was fixed to $61 \mathrm{~mm}$, as refined in the azimuthal integration step. The absorption coefficient at $6.5 \mathrm{keV}$ was calculated as $800 \mathrm{~cm}^{-1}$ (for a packing density of 0.8 , measured from $\mathrm{x}$ ray transmission measurement). These parameters were fixed for the refinements against time resolved patterns. The refinements included two phases ( $\beta$ - and $\lambda-$-). For each phase, the free parameters were the cell parameters (a, b, c, $\phi$ ), the atomic position in the $(\boldsymbol{a}, \boldsymbol{c})$ plane for the five independent atoms (position along $\boldsymbol{b}$ being symmetry-restricted) and the scale factor. An additional microstrain-type gaussian convolution (FWHM $=\varepsilon \times \tan (\theta)$ ) accounted for the strain distribution observed during the propagation (Fig. 5). The initial Bragg peak profile was well defined by the experimental resolution function defined as described above. Thus no extra size contribution was considered in the refinement of the reference patterns. The transformation being reversible and isostructural, no evolution of the crystallite size was expected and thus considered either. In any case, the choice was made to retain the simplest convolution functions to ensure the robustness of the refinement. Hence no lorentzian contribution, asymmetry or anisotropy was taken into account. The background was described as a 3rd order polynomial. The texture of the majority phase was described using Spherical Harmonics corrections, whose coefficients were refined on reference patterns and then kept constant for all delays. The $R_{\mathrm{wp}}$ agreement factors were around $7.5 \% ; R_{\mathrm{Bragg}}$ were calculated around $2 \%$ for the majority $\beta$ - phase and around $7 \%$ for the $\lambda$ - phase. For the SwissFEL data refinement, errors were estimated from the distribution (standard deviation $S D$ ) of the refined values obtained on reference pattern (interleaves data with no laser), and thus have to be understood as relative errors; the errors given in the text and in the figures corresponds to $2 \times S D$. Note that for the ESRF data, the quality of the extracted patterns did not allow to refine the atomic positions. In both cases, reference structures were taken from ${ }^{13,14}$. The atomic displacement parameters could never be refined accurately due to the limited $q$ region measured and large beam footprint resulting in poor $q$-resolution. These values were fixed with those reported in the literature.

\section{Data availability}

Raw data for the SwissFEL experiment, scripts to process them and to produce the final figures of the main text have been uploaded to the Zenodo repository and can be found here: https://doi.org/10.5281/zenodo.4327604. All other data can be provided upon reasonable request.

Received: 22 April 2020; Accepted: 13 January 2021; Published online: 23 February 2021 


\section{References}

1. Fleming, G. \& Ratner, M. Grand challenges in basic energy sciences. Phys. Today 61, 28 (2008).

2. K. Nasu, (ed.) Photoinduced Phase Transitions (World Scientific, 2004). https://doi.org/10.1142/5476

3. Yonemitsu, K. \& Nasub, K. Theory of photoinduced phase transitions in itinerant electron systems. Phys. Rep. 465, 1 (2008).

4. Zhang, J. \& Averitt, R. D. Dynamics and control in complex transition metal oxides. Annu. Rev. Mater. Res. 44, 19 (2014).

5. Basov, D. N., Averitt, R. D. \& Hsieh, D. Towards properties on demand in quantum materials. Nat. Mater. 16, 1077-1088 (2017).

6. Johnson, S. L. et al. Watching ultrafast responses of structure and magnetism in condensed matter with momentum-resolved probes. Struct. Dyn. 4, 061506 (2017).

7. Beaud, P. et al. A time-dependent order parameter for ultrafast photoinduced phase transitions. Nat. Mater. ume 13, 923 (2014).

8. Bertoni, R. et al. Elastically driven cooperative response of a molecular material impacted by a laser pulse. Nat. Mater. 15, 606 (2016)

9. Okimoto, Y. et al. Ultrasonic propagation of a metallic domain in $\mathrm{Pr}_{0.5} \mathrm{Ca}_{0.5} \mathrm{CoO}_{3}$ undergoing a photoinduced insulator-metal transition. Phys. Rev. Lett. 103, 027402 (2009).

10. Wang, X. et al. Generation of a large compressive strain wave in graphite by ultrashort-pulse laser irradiation. Struct. Dyn. 6, 024501 (2019).

11. Thomsen, C., Grahn, H. T., Maris, H. J. \& Tauc, J. Surface generation and detection of phonons by picosecond light pulses. Phys. Rev. B 34, 4129-4138 (1986).

12. Schick, D. et al. Ultrafast lattice response of photoexcited thin films studied by X-ray diffraction. Struct. Dyn. 1, 064501 (2014).

13. Onoda, M. Phase transitions of $\mathrm{Ti}_{3} \mathrm{O}_{5}$. J. Solid State Chem. 136, 67 (1998).

14. Ohkoshi, S. et al. Synthesis of a metal oxide with a room-temperature photoreversible phase transition. Nat. Chem. 2, 539 (2010).

15. Zacharias, M., Rosch, A. \& Gars, M. Critical elasticity at zero and finite temperature. Eur. Phys. J. Spec. Top. 224, 1021-1040 (2015).

16. Tokoro, H. et al. External stimulation-controllable heat-storage ceramics. Nat. Commun. 6, 7037 (2015).

17. Kobayashi, K. et al. Electronic structure and correlation in $\beta-\mathrm{Ti}_{3} \mathrm{O}_{5}$ and $\lambda$ $\mathrm{Ti}_{3} \mathrm{O}_{5}$ studied by hard $\mathrm{x}$-ray photoelectron spectroscopy. Phys. Rev. B 95, 085133 (2017).

18. Wang, M. et al. Phase evolution and formation of $\lambda$ phase in $\mathrm{Ti}_{3} \mathrm{O}_{5}$ induced by magnesium doping. J. Alloy. Compd. 774, 1189 (2019).

19. Shen, Z. et al. Stabilization of microcrystal $\lambda-\mathrm{Ti}_{3} \mathrm{O}_{5}$ at room temperature by aluminum-ion doping. Appl. Phys. Lett. 111, 191902 (2017).

20. Takahama, R. et al. Structural, magnetic, transport, and thermoelectric properties of the pseudobrookite $\mathrm{AlTi}_{2} \mathrm{O}_{5}-\mathrm{Ti}_{3} \mathrm{O}_{5}$ system. Phys. Rev. Mater. 4, 074401 (2020).

21. Ohkoshi, S. et al. Low-pressure-responsive heat-storage ceramics for automobiles. Sci. Rep. 9, 13203 (2019).

22. Asahara, A., Watanabe, H., Tokoro, H., Ohkoshi, S. \& Suemoto, T. Ultrafast dynamics of photoinduced semiconductor-to-metal transition in the optical switching nano-oxide $\mathrm{Ti}_{3} \mathrm{O}_{5}$. Phys. Rev. B 90, 014303 (2014).

23. Ould-Hamouda, A., Tokoro, H., Ohkoshi, S. \& Freysz, E. Single-shot time resolved study of the photo-reversible phase transition induced in flakes of $\mathrm{Ti}_{3} \mathrm{O}_{5}$ nanoparticles at room temperature. Chem. Phys. Lett. 608, 106 (2014)

24. Tasca, K. R. et al. Time-resolved X-ray powder diffraction study of photoinduced phase transitions in $\mathrm{Ti}_{3} \mathrm{O}_{5}$ nanoparticles. Chem. Phys. Chem. 18, 1385 (2017).

25. Ingold, G. et al. Experimental station Bernina at SwissFEL: condensed mand time scales investigated by X-ray diffraction and spectroscopic methods. J. Synchrotron Radiat. 26, 874 (2019).

26. Hakoe, F., Tokoro, H. \& Ohkoshi, S. Dielectric and optical constants of $\lambda$ $\mathrm{Ti}_{3} \mathrm{O}_{5}$ film measured by spectroscopic ellipsometry. Mater. Lett. 188, 8 (2017).

27. Abreu, E. et al. Dynamic conductivity scaling in photoexcited $\mathrm{V}_{2} \mathrm{O}_{3}$ thin films. Phys. Rev. B 92, 085130 (2015).

28. Ruello, P. \& Gusev, V. E. Physical mechanisms of coherent acoustic phonons generation by ultrafast laser action. Ultrasonics 56, 35 (2015).

29. Osamu Matsuda and Maria Cristina Larciprete, Voti, RobertoLi \& Wright, OliverB. Fundamentals of picosecond laser ultrasonics. Ultrasonics 56, 3-20 (2015).

30. Thomsen, C., Grahn, H. T., Maris, H. J. \& Tauc, J. Picosecond interferometric technique for study of phonons in the Brillouin frequency range. Opt. Commun. 60, 55 (1986).
31. Singer, A. et al. Nonequilibrium phase precursors during a photoexcited insulator-to-metal transition in $\mathrm{V}_{2} \mathrm{O}_{3}$. Phys. Rev. Lett. 120, 207601 (2018)

32. Cammarata, M. et al. Chopper system for single pulse experiments with synchrotron radiation. Rev. Sci. Instrum. 80, 015101 (2009).

33. Först, $M$. et al. Spatially resolved ultrafast magnetic dynamics initiated at a complex oxide heterointerface. Nat. Mater. 14, 883-889 (2015).

34. Först, M. et al. Multiple supersonic phase fronts launched at a complex-oxide heterointerface. Phys. Rev. Lett. 118, 027401 (2017).

35. Ashiotis, G. et al. The fast azimuthal integration Python library: pyFAI. J. Appl. Crystallogr. 48, 510 (2015).

36. Harmand, M. et al. Achieving few-femtosecond time-sorting at hard X-ray free-electron lasers. Nat. Photonics 7, 215 (2013).

37. Mozzanica, A. et al. The JUNGFRAU detector for applications at synchrotron light sources and XFELs. Synchrotron Radiat. N. 31, 16 (2018).

38. Coelho, A. A. TOPAS and TOPAS-Academic: an optimization program integrating computer algebra and crystallographic objects written in $\mathrm{C}++$. J. Appl. Cryst. 51, 210 (2018).

39. Rowles, M. R. \& Buckley, C. E. Aberration corrections for nonBragg-Brentano diffraction geometries. J. Appl. Cryst. 50, 240 (2017).

\section{Acknowledgements}

The authors wish to thank Gemma Newby and Martin Pedersen from ESRF for support during the early phase of this project, Guénolé Huitric (IPR) for his contribution to the optical spectroscopy measurements performed at IPR as discussed in the SI. M.C. wants to thank PSI for accepting his proposal to study $\mathrm{Ti}_{3} \mathrm{O}_{5}$ nanoparticles as the first experiment for the entire facility and for providing excellent support. M.Lo gratefully acknowledges the Agence Nationale de la Recherche for financial support under grant ANR-16-CE30-0018 ("Elastica"). B.L. would like to thank Bruno Bêche (IPR) for help with AFM measurement. M.C. and M.Le also wish to acknowledge the support from the European Union Horizon 2020 under the Marie Sklodowska-Curie Project "X-Probe" Grant No. 637295. S.O. and H.T. acknowledge the support from JSPS Grant-in-Aid Grant Number 15H05697, Scientific Research(A) 20H00369, and JSPS KAKENHI Grant Number 16H06521 Coordination Asymmetry. M.Ch and G.F.M. acknowledge the funding from the European Research Council (ERC) H2020 DYNAMOX (grant No 695197 and from the Swiss NSF via the NCCR:MUST). Parts of this research were carried out in the frame of the IM-LED LIA (CNRS).

\section{Author contributions}

C.M., M.Lo, H.C., and M.C. coordinated the project. H.C. and M.Lo proposed the study of the photoinduced phase transition of $\mathrm{Ti}_{3} \mathrm{O}_{5}$. M.C. coordinated the X-ray studies. C.M. developed the thermo-elastic model with the help of M.Lo. Nanocrystals of $\mathrm{Ti}_{3} \mathrm{O}_{5}$ have been characterized and synthesized by S.O. and H.T. C.M. and L.C. performed Rietveld refinement with help from O.H. O.H. performed the static powder XRD measurements. A.Bo. performed diffuse scattering measurements and high resolution powder diffraction. M.C. and M.Le developed the setup at ESRF with help of M.W., S.Z., E.T., E.C., X.D., C.M., M.Lo. M.C. and H.L. developed the setup at SwissFEL with help from L.G., A.V., C.M., M.Le, L.P., P.Be., V.E., Y.D., G.I., G.M., R.M., C.S., S.Z., I.U.; D.O. and L.S helped with the data acquisition; S.E., P.Bo., A.K., A.O., T.Z., S.V., A.Ba., R.F., P.J. developed Bernina components and helped assembling them. M.C. developed the data reduction procedure. A.M. developed the Jungfrau detector used for the experiment with help from S.R. V.T. performed DFT. calculations with help from E.J. C.M., M.Lo, H.C., and M.C. wrote the manuscript with significant contributions from E.C., E.J., L.C., M.Le P.B., critical reading from M.Ch and help from all authors.

\section{Competing interests}

The authors declare no competing interests.

\section{Additional information}

Supplementary information The online version contains supplementary material available at https://doi.org/10.1038/s41467-021-21316-y.

Correspondence and requests for materials should be addressed to C.M., M.Lo. or M.C.

Peer review information Nature Communications thanks Kenneth Beyerlein, Wanxia Huang, and other anonymous reviewers for their contributions to the peer review of this work. Peer review reports are available.

Reprints and permission information is available at http://www.nature.com/reprints

Publisher's note Springer Nature remains neutral with regard to jurisdictional claims in published maps and institutional affiliations. 
(c) (i) Open Access This article is licensed under a Creative Commons Attribution 4.0 International License, which permits use, sharing, adaptation, distribution and reproduction in any medium or format, as long as you give appropriate credit to the original author(s) and the source, provide a link to the Creative Commons license, and indicate if changes were made. The images or other third party material in this article are included in the article's Creative Commons license, unless indicated otherwise in a credit line to the material. If material is not included in the article's Creative Commons license and your intended use is not permitted by statutory regulation or exceeds the permitted use, you will need to obtain permission directly from the copyright holder. To view a copy of this license, visit http://creativecommons.org/ licenses/by/4.0/.

(C) The Author(s) 2021 